\title{
ON SPACES WHICH ARE ESSENTIALLY $T_{1}$
}

\author{
DICK WICK HALL, SHEILA K. MURPHY and EUGENE P. ROZYCKI
}

(Received 31 July 1969)

Communicated by E. Strzelecki

Let $S$ be a pseudo-metric space with pseudo-metric $d$. Then for each nonempty $A \subseteq S, x \in \operatorname{cl}(A)$ if and only if $d(x, A)=0$, and $d(x, y)=d(y, x)$ for all $x, y \in S$. Thus, if $d(x, y)=0$, then $x \in \operatorname{cl}(\{y\})$ by the first requirement and $y \in \operatorname{cl}(\{x\})$ by the second requirement. It is natural then to expect that if a topological space $S$ is to be pseudo-metrizable, it should at least satisfy the requirement:

$$
\text { If } x, y \in S \text { and } x \in \operatorname{cl}(\{y\}) \text {, then } y \in \operatorname{cl}(\{x\}) .
$$

Spaces satisfying this requirement were termed essentially $T_{1}$ by Worrell and Wicke [6] and $R_{0}$ by Davis [1]. Several characterizations have been given for such spaces by Davis (ibid.). In this paper we propose to give another characterization and to use these characterizations to indicate various classes of spaces that have this property built into their definition, a fact which apparently has been generally unnoticed. Although the concept of being an essentially $T_{1}$ space is certainly modest in scope, we hope to indicate further the role this property plays in topology and that the results of our investigations about this role will be of interest.

It is easy to see that if such a space is also $T_{0}$, then it is necessarily $T_{1}$. Thus $T_{1}$ spaces are those which are both $T_{0}$ and essentially $T_{1}$. Since there are $T_{0}$ spaces which are not $T_{1}$ and every pseudo-metric space which is not metric is essentially $T_{1}$ but not $T_{0}$, the properties of a space being $T_{0}$ and essentially $T_{1}$ are independent of one another. Moreover, this shows that for spaces which are inherently essentially $T_{1}$, it is necessary to assume only the $T_{0}$ separation property whenever the $T_{1}$ separation property is desired. A simple example of this is the following:

A space $S$ is metric if and only if it is pseudo-metric and $T_{0}\left(\right.$ rather than $\left.T_{1}\right)$.

In a pseudo-metric space, every closed set can be expressed as a countable intersection of open sets. Such sets are termed $G_{\boldsymbol{b}}$. The following theorem shows that essentially $T_{1}$ spaces may be considered as generalizations of spaces all of whose closed sets have this property.

THEOREM 1. The following statements about a space $S$ are equivalent:

(a) $S$ is essentially $T_{1}$. 
(b) For every pair of points $x$ and $y$ of $S$, the sets $\mathrm{cl}(\{x\})$ and $\mathrm{cl}(\{x\})$ are either equal or disjoint.

(c) Every open set contains the closure of each of its points.

(d) Every open set is the union of some collection of closed sets of $S$.

(e) Every closed set is the intersection of some collection of open sets of $S$.

Proof. (a) implies (b): Let $x, y \in S$, and suppose there is a point $z \in \operatorname{cl}(\{x\}) \cap$ $\operatorname{cl}(\{y\})$. Since $\mathrm{cl}(\{x\}) \cap \mathrm{cl}(\{y\})$ is a closed set containing $\{z\}$, we have $\mathrm{cl}(\{z\}) \subseteq$ $\operatorname{cl}(\{x\}) \cap \operatorname{cl}(\{y\})$. Now $z \in \operatorname{cl}(\{x\})$ implies $x \in \operatorname{cl}(\{z\})$, and so $\operatorname{cl}(\{x\}) \subseteq \operatorname{cl}(\{z\})$. Similarly $\operatorname{cl}(\{y\}) \subseteq \operatorname{cl}(\{z\})$. Thus $\operatorname{cl}(\{x\}) \cup \operatorname{cl}(\{y\})=\operatorname{cl}(\{y\}) \cap \operatorname{cl}(\{y\})$ and we conclude that $\operatorname{cl}(\{x\})=\operatorname{cl}(\{y\})$.

(b) implies (c): Let $x \in U$ where $U$ is open, and let $y \in \operatorname{cl}(\{x\})$. By (b), $\operatorname{cl}(\{x\})=\operatorname{cl}(\{y\})$, hence $x \in \operatorname{cl}(\{y\})$. Since $U$ is an open set containing $x$, we must have that $y \in U$. Thus $\operatorname{cl}(\{x\}) \subseteq U$.

(c) implies (d): Let $U$ be a non-empty open set of $S$. By (c), $U=\bigcup_{x \in U} \mathrm{cl}(\{x\})$.

(d) implies (e): This follows immediately by taking complements.

(e) implies (a): Let $x, y \in S$ and $x \in \operatorname{cl}(\{\} y)$. Then every open set containing $x$ must contain $y$. Suppose $y \notin \operatorname{cl}(\{x\})$. Then by (e), some open set containing $\operatorname{cl}(\{x\})$, and hence $x$, does not contain $y$, a contradiction.

The statement that the space $S$ is a developable space means that there is a sequence $G_{1}, G_{2}, \cdots$ of collections of open sets such that for each $i, G_{i}$ covers $S, G_{i+1} \subseteq G_{i}$, and if $x \in U$ where $U$ is open, there is an $i$ such that $x \in V \in G_{i}$ implies $V \subseteq U$. The sequence $G_{1}, G_{2}, \cdots$ is called a development for $S$.

The statement that the space $S$ is a pseudosemi-metric space means that there exists a non-negative, real-valued function $d$, defined on $S \times S$ and such that (i) $d(x, x)=0$ for all $x \in S$; (ii) $d(x, y)=d(y, x)$ for all $x, y \in S$; and (iii) for each non-empty $A \subseteq S, x \in \operatorname{cl}(A)$ if and only if $d(x, A)=0$. If, in addition, we have that $d(x, y)=0$ implies $x=y$, then $S$ is termed a semi-metric space.

It follows immediately from the definition of a pseudosemi-metric space, that it is necessarily essentially $T_{1}$. Since every developable space is a pseudosemimetric space (Heath [3]), it follows that every developable space is essentially $T_{1}$. Just as for metric and pseudo-metric spaces, we have:

A space $S$ is a semi-metric space if and only if it is a pseudosemi-metric space and $T_{0}$.

Another interesting and important class of spaces that are essentially $T_{1}$ is given by the following theorem, a proof of which may be found in Thron ([5], page 98). The $T_{1}$ separation property is not being assumed here.

THEOREM 2. Every regular space is essentially $T_{1}$.

In particular, this shows that every uniform space is essentially $T_{1}$ since each such space is completely regular. 
Normal spaces are not necessarily essentially $T_{1}$. This is illustrated by the simple example where $S=\{a, b, c\}$ and the open sets are $\emptyset,\{a\},\{b\},\{a, b\}$, and $S$.

The relation, $x R y$ if and only if $\operatorname{cl}(\{x\})=\operatorname{cl}(\{y\})$ for an arbitrary space $S$, is an equivalence relation such that the resulting space $S / R$ is $T_{0}$ (Pervin [4], page 155) and whose topology is lattice isomorphic to that of the space $S$ (Thron [5], page 92). If the space $S$ is also essentially $T_{1}$, the equivalence classes induced by $R$ are of the form $\mathrm{cl}(\{x\})$ and the resulting quotient space is necessarily $T_{1}$. From this and a result by Finch ([2], corollary $\left.(2.1)^{\prime}\right)$, it follows that if $S_{1}$ and $S_{2}$ are essentially $T_{1}$ spaces and $R_{1}, R_{2}$ are relations defined as above for $S_{1}, S_{2}$, respectively, then $S_{1} / R_{1}$ and $S_{2} / R_{2}$ are lattice isomorphic if and only if $S_{1} / R_{1}$ and $S_{2} / R_{2}$ are homeomorphic. From this there follows:

THEOREM 3. If $S_{1}$ and $S_{2}$ are essentially $T_{1}$ spaces, then $S_{1}$ and $S_{2}$ are lattice isomorphic if and only if $S_{1} / R_{1}$ and $S_{2} / R_{2}$ are homeomorphic.

In the discussion immediately preceding the above theorem it was pointed out that the identification process $x \rightarrow \operatorname{cl}(\{x\})$ yielded a $T_{1}$ space if the original space was essentially $T_{1}$. It is well-known that if $S$ is a pseudo-metric space with pseudometric $d$, then the resulting quotient space $S / R$ is a metric space with metric $D$ defined by $D(\operatorname{cl}(\{x\}), \operatorname{cl}(\{y\}))=d(x, y)$ with the map $x \rightarrow \operatorname{cl}(\{x\})$ being distancepreserving. The only serious problem one encounters in proving this theorem is to show that the function $D$ is well defined and this is easily overcome by application of the triangle inequality to the pseudometric $d$. Since this property is no longer available for pseudosemi-metrics, it would be of interest to determinee whether the space $S / R$ is a semi-metric space when $S$ is a pseudosemi-metric space. That this is indeed the case follows from a modified result of Heath [3]. He introduced three conditions which lead from a $T_{1}$ space to a metric space, with semi-metric and developable spaces as intermediate steps. If one is willing to replace points by their closures, the $T_{1}$-separation property may be dropped and the same conditions will lead from an arbitrary space to a pseudo-metric space, with pseudosemi-metric and developable spaces as intermediate steps.

DEFINITION. Let $S$ be a space such that for each point $x \in S$, there exists a sequence $\left\langle G_{i}(x)\right\rangle$ of open sets, such that $x \in G_{i}(x) \subseteq G_{i-1}(x)$ for each $i>1$.

Condition A. For each $x \in S,\left\{G_{i}(x) \mid i=1,2, \cdots\right\}$ is a local base at $x$. If $y \in S$, and if $\left\langle x_{n}\right\rangle$ is a sequence of points of $S$, such that for each $n, y \in G_{n}\left(x_{n}\right)$, then $\left\langle x_{n}\right\rangle$ converges to $y$.

CONDITION B. If $y \in S$, and $\left\langle x_{n}\right\rangle$ and $\left\langle z_{n}\right\rangle$ are sequences of points of $S$, such that for each $n,\left\{y, x_{n}\right\} \subseteq G_{n}\left(z_{n}\right)$, then $\left\langle x_{n}\right\rangle$ converges to $y$.

Condition C. If $x, y \in S$, and there is an $n$, such that $x \in G_{n}(y)$, then $y \in G_{n}(x)$.

We first make the observation 
THEOREM 4. If $S$ is a space satisfying Condition A, then every closed subset of $S$ is $a G_{\delta}$. Thus, $S$ is essentially $T_{1}$.

Proof. Let $F \subseteq S$ be closed. For each $i$, let $S_{i}(F)=\bigcup\left\{G_{i}(x) \mid x \in F\right\}$. Then $F \subseteq S_{i}(F)$ and $S_{i}(F)$ is open for each $i$. Hence $F \subseteq \bigcap_{i} S_{i}(F)$. If $x \in \bigcap_{i} S_{i}(F)$, then for each $i$, there is a point $x_{i} \in F$, such that $x \in G_{i}\left(x_{i}\right)$. Then $\left\langle x_{i}\right\rangle$ converges to $x$, and so every neighborhood of $x$ contains a point $x_{i}$ of $F$. Thus $x \in \mathrm{cl}(F)=F$ and $F=\bigcap_{i} S_{i}(F)$.

THEOREM 5. (a) A space $S$ is a pseudosemi-metric space if and only if $S$ satisfies Condition $A$.

(b) A space $S$ is developable if and only if $S$ satisfies Condition $B$.

(c) A space $S$ is pseudometrizable if and only if $S$ satisfies Conditions $B$ and $C$.

We shall not give the proof here since it is rather lengthy but mention that its basic outline is contained in Heath [3].

A result that rather surprised us, and which together with Theorem 5 above, gives another proof that every developable space is pseudosemi-metric, is the following:

Theorem 6. If a space $S$ satisfies Condition $B$, then $S$ satisfies Condition $A$.

Proof. Let $x \in S$. We must show that $\left\{G_{i}(x) \mid i=1,2, \cdots\right\}$ is a local base at $x$. For, if not, then there is a neighborhood $U$ of $x$, and for each $n$, a point $y_{n} \in G_{n}(x)$ such that $y_{n} \notin U$. Then $\left\{x, y_{n}\right\} \subseteq G_{n}(x)$, and using Condition B with $z_{n}=x$ for each $n$, then $\left\langle y_{n}\right\rangle$ converges to $x$, and thus $y_{n} \in U$ for all but finitely many $n$, a contradiction. Hence $\left\{G_{i}(x) \mid i=1,2, \cdots\right\}$ is a local base at $x$. Finally, let $y \in S$ and $\left\langle x_{n}\right\rangle$ a sequence of points, such that for each $n, y \in G_{n}\left(x_{n}\right)$. Then $\left\{y, x_{n}\right\} \subseteq G_{n}\left(x_{n}\right)$ for each $n$, and by Condition $\mathbf{B},\left\langle x_{n}\right\rangle$ converges to $y$. Condition $\mathrm{A}$ is thus satisfied.

In the following let $R$ be the equivalence relation which identifies closures of points.

THEOREM 7. (a) If $S$ is a pseudosemi-metric space, then $S / R$ is a semi-metric space.

(b) If $S$ is a developable space, then $S / R$ is also a developable space.

Proof. We give a proof of (a) only, the proof of (b) being similar. The projection map $p: S \rightarrow S / R$ is open, closed, and continuous. Well-order the points of $S$. By Theorem 5(a), $S$ satisfies Condition A. Let the elements of $S / R$ be dedenoted by $R[x]$, where $x \in S$. Given $R[x] \in S / R$, for each $n$, define $g_{n}(R[x])=$ $p\left(G_{n}(z)\right)$, where $z$ is the first point of $S$ in $\operatorname{cl}([x])$. Then $g_{n}(R[x])$ is an open set of $S / R$. Also, if $n>1, z \in G_{n}(z) \subseteq G_{n-1}(z)$ implies $R[x]=R[z] \in p\left(G_{n}(z)\right) \subseteq$ $p\left(G_{n-1}(z)\right)$. Thus $R[x] \in g_{n}(R[x]) \subseteq g_{n-1}(R[x])$.

To show that $\left\{g_{i}(R[x]) \mid i=1,2, \cdots\right\}$ is a local base at $R[x]$, let $U$ be any neighborhood of $R[x]$ in $S / R$. Then $p^{-1}(U)$ is open and contains $\mathrm{cl}(\{x\})$, hence 
$z \in p^{-1}(U)$, where $z$ is again the first element in $\operatorname{cl}(\{x\})$. There exists an $n$ then, such that $G_{n}(z) \subseteq p^{-1}(U)$. But then, $g_{n}(R[x])=p\left(G_{n}(z)\right) \subseteq p\left(p^{-1}(U)\right)=U$, and so $\left\{g_{i}(R[x]) \mid i=1,2, \cdots\right\}$ is a local base at $R[x]$.

Given $R[y] \in S / R$, and a sequence, $\left\langle R[x]_{n}\right\rangle$, of points of $S / R$, such that for each $n, R[y] \in g_{n}\left(R[x]_{n}\right)$, let $t$ be the first point of $\operatorname{cl}(\{x\})$, and $z_{n}$ the first point of $p^{-1}\left(R[x]_{n}\right)$. Then for each $n, t \in \operatorname{cl}(\{y\}) \subseteq p^{-1}\left(p\left(G_{n}\left(z_{n}\right)\right)\right)=G_{n}\left(z_{n}\right)$. Since $S$ satisfies Condition $\mathrm{A},\left\langle z_{n}\right\rangle$ converges to $t$. Let $U$ be any neighborhood of $R[y]$. Then $p^{-1}(U)$ is open and contains $\operatorname{cl}(\{y\})$, hence $t \in p^{-1}(U)$. Then there is an $m$, such that $z_{n} \in p^{-1}(U)$ for all $n \geqq m$. So, $p\left(z_{n}\right)=R[x]_{n} \in p\left(p^{-1}(U)\right)=U$ for all $n \geqq m$, and $\left\langle R[x]_{n}\right\rangle$ converges to $R[y]$. Therefore, $S / R$ satisfies Condition $\mathrm{A}$, and since $S / R$ is $T_{1}, S / R$ is a semi-metric space.

\section{References}

[1] A. S. Davis, 'Indexed systems of neighborhoods for general topological spaces', Amer. Math. Monthly 68 (1961), 886-893.

[2] P. D. Finch, 'On the lattice equivalence of topological spaces', J. Austral. Math. Soc. 6 (1966), 495-511.

[3] R. W. Heath, 'Arcwise connectedness in semi-metric spaces', Pacific J. Math. 12 (1962), $1301-1319$.

[4] W. J. Pervin, Foundations of General Topology (Academic Press, New York, 1964).

[5] W. J. Thron, Topological Structures (Holt, Rinehart, and Winston, New York, 1966).

[6] J. M. Worell, Jr., and H. H. Wicke, 'Characterizations of developable topological spaces, Can. J. Math. 17 (1965), 820-830.

State University of New York at Binghamton 